AML-HEP-CP--83-54

\title{
STUDY OF QUARK FRAGMENTATION AT 29 GeV*
}

\author{
HRS COLlaboration \\ Presented by M. Valdata-Nappi \\ Argonne National Laboratory
}

S. Ahlen, C. Akerlof, G. Baranko, I. Beltrami, D. Bender, P. Baringer, D. Blockus, B. Brabson, J. Chapman, B. Cork, R. DeBonte, M. Derrick, D. Errede, E. Fernandez, G.E. Forden, R. Fries, K.K. Gan, G. Gieraltowski, S.W. Gray, J.P. Guillaud, N. Harnew, L. Hyman, K. Jaeger, L. Keller, P. Kesten, R. Klem, D. Koltick, P. Kooijman, S. Kooijman, F. Loeffler, J.S. Loos, F. LoPinto, U. Mallik, R. Mcllwain, D.I. Meyer, D.H. Miller, B. Musgrave, M. Valdata-Nappi, H. Neal, R. Ng, D. Nitz, H. Ogren, P.P. Ong, L.E. Price, L. Rangan, D. Rubin, D. Rust, P. Schreiner, E.I. Shibata, A.A. Seidl, J. Schlereth, R. Singer, F.R. Stevens, R. Thun, T. Trinko, J. Va'vra, C. Ward, J.M. Weiss, M. Willutzky, R.J. Wilson, and D.E. Wood

Argonne National Laboratory, Argonne, Illinois 60499

Indiana University, Bloomington, Indiana 17401

Lawrence Berkeley Lajoratory, Berkekey, California 94720

University of Michigan, Ann Arbor, Michigan 48109

Purdue University, West Lafayette, Indiana 47907

and

Stanford Linear Accelerator Center

Stanford University, Stanford, California 94905

"Work supported by the Department of Energy, contract DE-AC03-76SF00515.

Presented at the

XIV Interntional Symposium on Multiparticle Dynamics

Lake Tahoe, California, June 1983 


\section{DISCLAIMER}

This report was prepared as an account of work sponsored by an agency of the United States Government. Neither the United States Government nor any agency thereof, nor any of their employees, makes any warranty, express or implied, or assumes any legal liability or responsihility for the accuracy, completeness, or usefulness of any information, apparatus, product, or process disclosed, or represents that its use would not infringe privately owned rights. Reference herein to any specific commercial product, process, or service by trade name, trademark, manufacturer, or otherwise does not necessarily constitute or imply its endorsement, recommendation, or favoring by the United States Government or any agency thereof. The views and opinions of authors expressed herein do not necessarily state or reflect those of the United Siates Government or any agency thereof. 


\section{Introduction}

The study of multi-particie final state in high-energy reactions has a long history. Detailed studies of hadronic interactions at Fermilab ${ }^{1)}$ and at the CERN ISR $^{2)}$ have shown that particles are emitted in clear jets of hadrons along the beam directions. For the highest energies, a small fraction of events contains large-angle jets originating from hard collisions, in addition to the beam and target fragmentation products ${ }^{3}$.

The clearest observation of jets has been in high-energy $e^{+} e^{-}$reactions. Hadronic interactions contain 5 or 6 valence quarks already in the initial state. The final state in $e^{+} e^{-}$annihilation represents at the parton level a relatively simple dynamical system: a $q \bar{q}$ state plus possibly a hard gluon ${ }^{4)}$ therefore jets produced in $e^{+} e^{-}$annihilation have to be considered as the cleanest ones. The study of their properties can help, not only to understand quark fragmentation but may also serve as an important tool in the analysis of the much more complex mechanism in hadronic interactions ${ }^{5}$.

In this paper we present preliminary results of the overall features of our first sample of $e^{+} e^{-}$annihilation events at $29 \mathrm{GeV}$.

The data were taken using the High Resolution Spectrometer (HRS) operated at the PEP storage ring at the Stanford Linear Accelerator Center.

\section{Description of the Apparatus and Event Selection}

The HRS, shown in Fig. 1, is a general purpose $e^{+} e^{-}$detector that provides excellent measurements of charged particle momenta.

The tracking is done in a $1.62 T$ magnetic field by a combination of a 15 layer inner drift chamber covering $80 \%$ of $4 \pi$ and 2 layers of drift tubes comprising an outer drift chamber which covers .62 of $4 \pi$. The momentum resolution for $14.5 \mathrm{GeV} / \mathrm{c}$ particles reaching the outer drift chamber is $\sigma_{p}=1.0 \times 10^{-3} p^{2}$ $(\mathrm{GeV})$, while the inner drift chamber alone measures momenta to a precision of $5.0 \times 10^{-3} p^{2}(\mathrm{GeV})$.

The tracking system is surrounded by lead scintillation sandwich electromagnetic calorimeters (barrel and end-cap). The energy resolution is $\sigma(E)=$ $.16 \sqrt{E}(E$ in $\mathrm{GeV})$ and $\sigma(E)=.20 \sqrt{E}$ for the barrel and end-caf, respectively. The position of the showering particle is determined by a set of proportional tubes. 


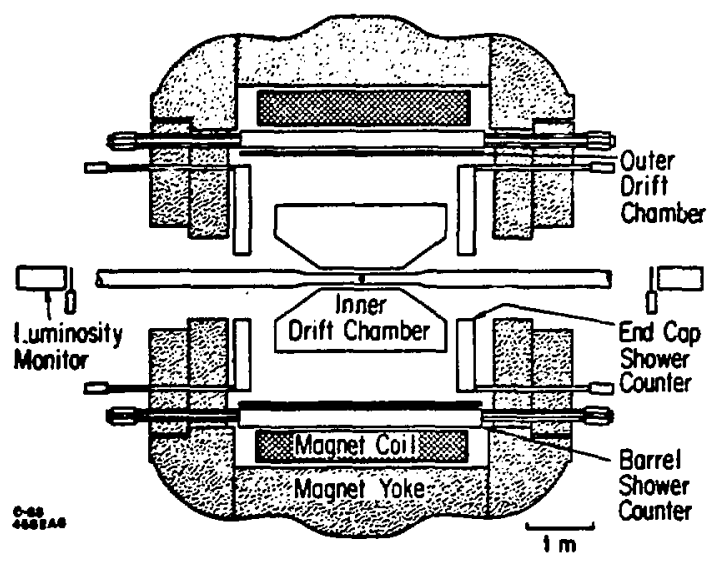

Fig. 1. Cross section view of the High Resolution Spectrometer.

The total detector thickness is .02Xo from the annibilation point to the outer tracking layer.

One photon annihilation events were triggered by the observation of $\geq 2$ tracks in the drift chamber or by the deposition of $\geq 4.8 \mathrm{GeV}$ of neutral energy in the calorimeters.

The data presented were obtained during 1981-1982 PEP running cycle and correspond to an integrated luminosity of $19.6 \mathrm{pb}^{-1}$. The normal PEP operating energy was $14.5 \mathrm{GeV}$ per beam, but a small fraction of the data were taken at 14.02 GeV per beam.

To eliminate beam-gas and two photon background the events selected for this analysis had $\geq \mathbf{5}$ charged prongs with a vertex constrained to the interaction point, a visible energy of $\Sigma|\vec{p}| \geq 8 \mathrm{GeV}$ and $\geq 2.0 \mathrm{GeV}$ of energy deposited in the calorimeters.

The selected tracks were required to extrapolate to the beam vertex and to have an emission angle $\geq 25^{\circ}$ io the beam axis. These cuts accepted most of the charged tracks from $K^{\circ}$ and $\Lambda$ decays. Minimum and maximum momentum values for any accepted track were pmin $=.1 \mathrm{GeV}$ and $p \max =15 \mathrm{GeV}$.

\section{Two Jet Events Selection}

Our two jet sample was selected and studied in terms of the jet variables sphericity $(S)$, aplanarity $(A)$ and thrust $(T)^{6,7)}$. 
In order to select a sample of $q \not{q}$ fragmentation events, we used the distribution of events in the sphericity-aplanarity space shown in Fig. 2. The definition of a two-jet event is arbitrary because gluon emission is a radiative process, but we choose $S \leq .25$ and $A \leq .1$ to define the two jet sample used in this paper.

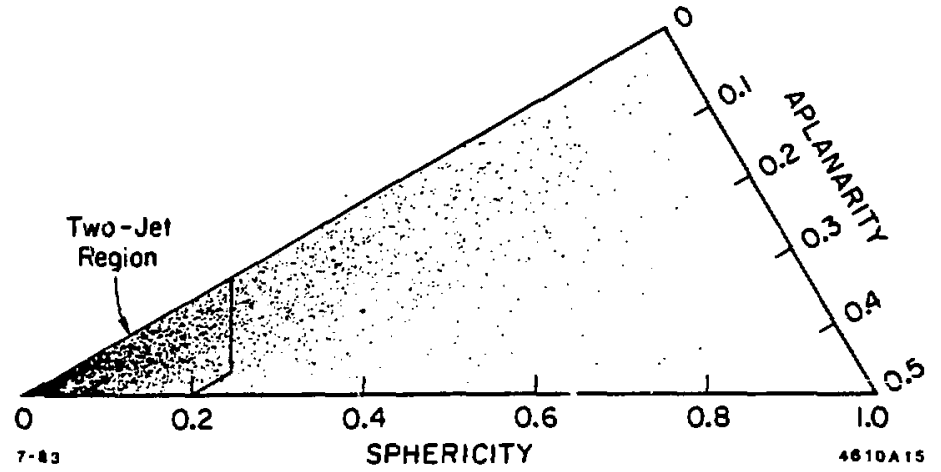

Fig. 2. Distribution of events in the sphericity-aplanarity space.

In the following we restrict our studies to the two jet sample and, since we will be concentrating on the charged particles, we further require that both jets have $\geq 2 \mathrm{GeV} / \mathrm{c}$ of charged particle momentum. The jet axis of the selected two jet sample is defined by the $T$ axis. In order to minimize any geometrical bias in the detector events were required to have the $T$ axis $\geq 45^{\circ}$ with respect to the beam direction. The net measured charge was required $\leq 4$ units.

With these selections we tagged 4183 events as two jet events. The distribution in azimuthal and polar angle of the $T$ axis is shown in Fig. 3, in good agreement with the expected distribution isotropic and $\left(1+\cos ^{2} \theta\right)$, respectively.

\section{Results}

\subsection{Multiplicity}

The multiplicity distribution of our data, shown in Fig. 4 as a fraction of the mean, as first suggested by Koba, Nielsen and Olesen ${ }^{8)}$ (KNO), is in good agreement with inclusive PETRA data'.

Our data are compared with the KNO distribution of $p p$ and $p \bar{p}$ annihilation data. Our results show a narrower distribution than the $p p$ data, while are similar to $p \bar{p}$ annihilation data. 

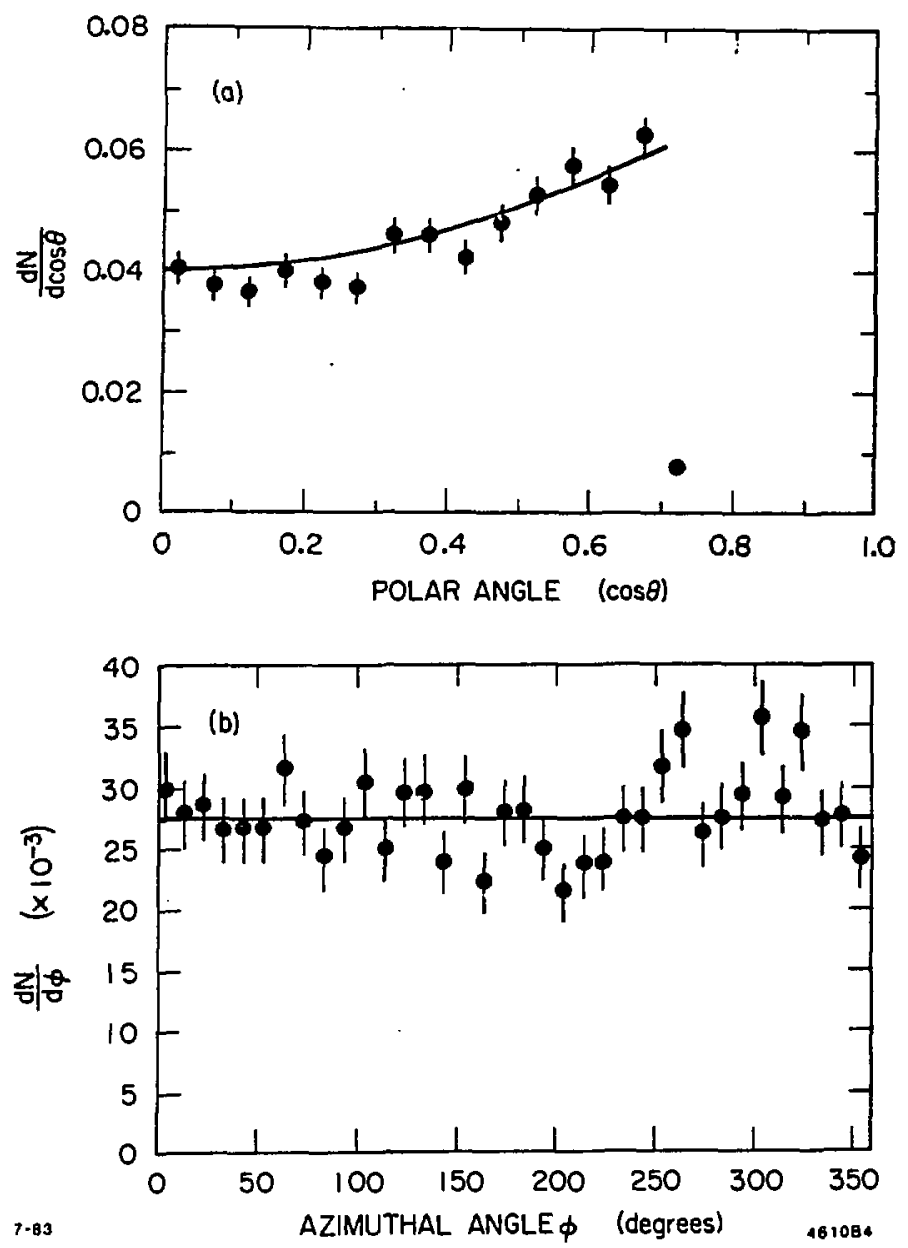

Fig. 3. Distribution of (a) polar angle $(\theta)$, (b) azimuthal angle $(\phi)$ of the thrust axis. The expected $1+\cos ^{2} \theta$ and isotropic $\phi$ are shown as the lines.

\subsection{TEST OF FRAGMENTATION INDEPENDENCE}

We have performed a few simple tests to understand to what extent the quark jets fragment independently. We assign the charged particles in a given event to one or the other jet, according to which detector hemisphere they populate; the equator is the plane perpendicular to the thrust axis. 


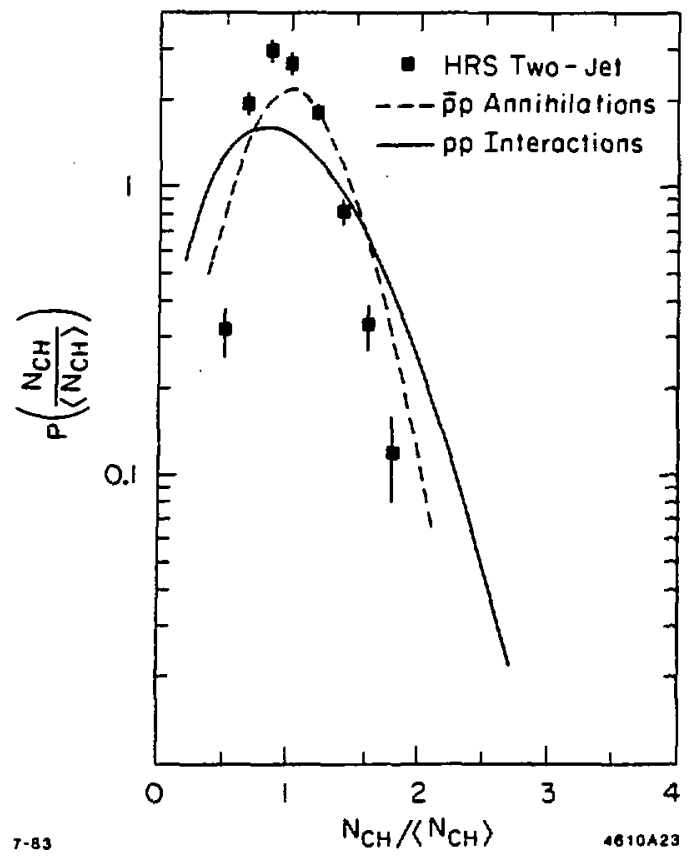

Fig. 4. KNO multiplicity distribution for the two jet sample compared to the Slattery fit to the $p p$ results ${ }^{10)}$ and $\bar{p} p$ annihilation data. ${ }^{11)}$

Figure 5 shows the distribution of the mean multiplicity in one jet $(F)$ compared to the number of charged particles in the second jet $(B)$. There is clearly not a strong correlation between the multiplicities in the two hemispheres. We have repeated the analysis (Fig. 5 b) with the central rapidity region $(|Y| \leq 1)$ removed. This selection minimizes possible biasses coming from particles in the central region whose jet assignment could be affected by the uncertainties of the thrust axis direction. It also removes the low momentum particles that are not reconstructed by the HRS with full efficiency.

The data are compared with a Monte-Carlo prediction using Field-Feynman fragmentation technique (dashed curve). ${ }^{12)}$ The agreement is good, supporting the idea of andependent fragme $s$. ttion of the two jets. Our data are also contrasted to the results of proton-proton collisions ${ }^{13}$ ) at $\sqrt{s}=31$ and $63 \mathrm{GeV}$ (full and dotted lines). While $e^{-1} e^{-}$annihilation data favor independent fragmentation, hadronic data show a positive correlation between the two hemispheres multiplicities. 


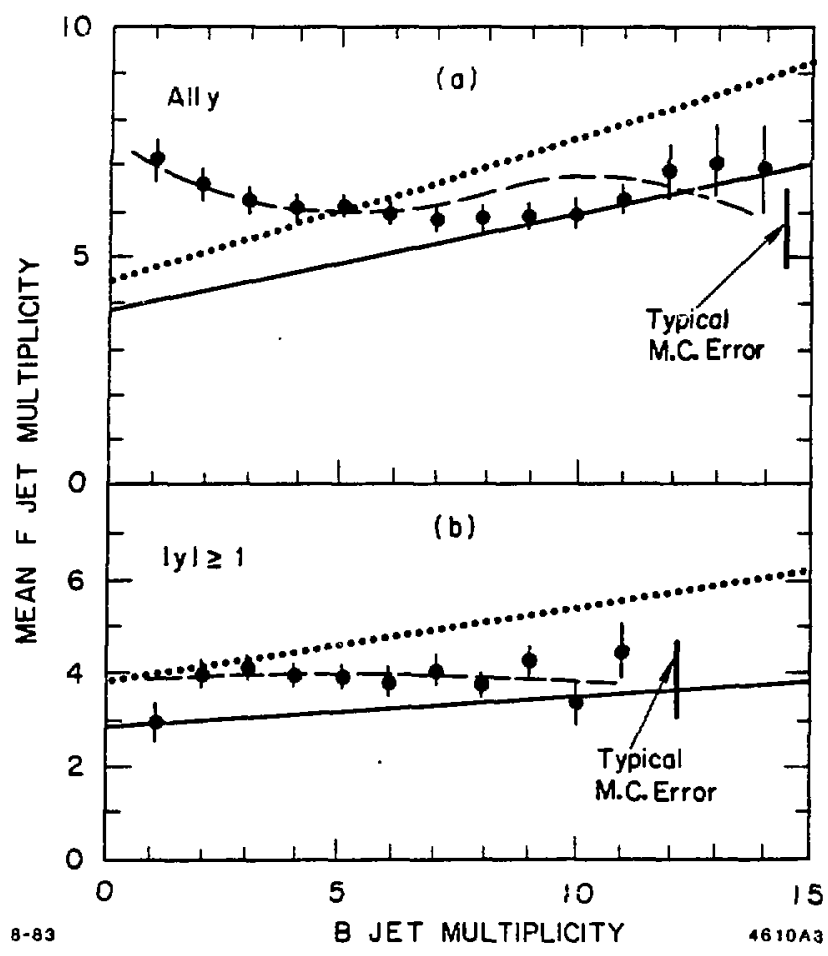

Fig. 5. Forward-backward multiplicity correlations (a) all data, (b) region $|y| \leq 1$ removed. Dashed lines indicate Monte Carlo with Field and Feynman parameterization; solid lines indicate hadronic ( $p p$ ) data at $\sqrt{s}=31 \mathrm{GeV}$; and dotted lines indicate hadronic ( $p p$ ) data at $\sqrt{8}=63 \mathrm{GeV}$.

The jet mass $(M)$ has been calculated using only the charged particles and assigning pion masses to the observed hadrons. The lack of a $F / B$ dependence of the jet mass of Fig. 6 further supports the idea of independent fragmentation of the $q$ and $\bar{q}$.

\subsection{Charge Compensation}

In Fig. 7 we show $\bar{\phi}\left(y_{1}, y_{2}\right)$ for a test region selection in the range $2.5 \leq$ $y_{2} \leq 5.5$ where:

$$
\phi\left(y_{1}, y_{2}\right)=\left(\rho^{+-}\left(y_{1}, y_{2}\right)+\rho^{-+}\left(y_{1}, y_{2}\right)\right)-\left(\rho^{++}\left(\left(y_{1}, y_{2}\right)+\rho^{--}\left(y_{1}, y_{2}\right)\right)\right.
$$

and 


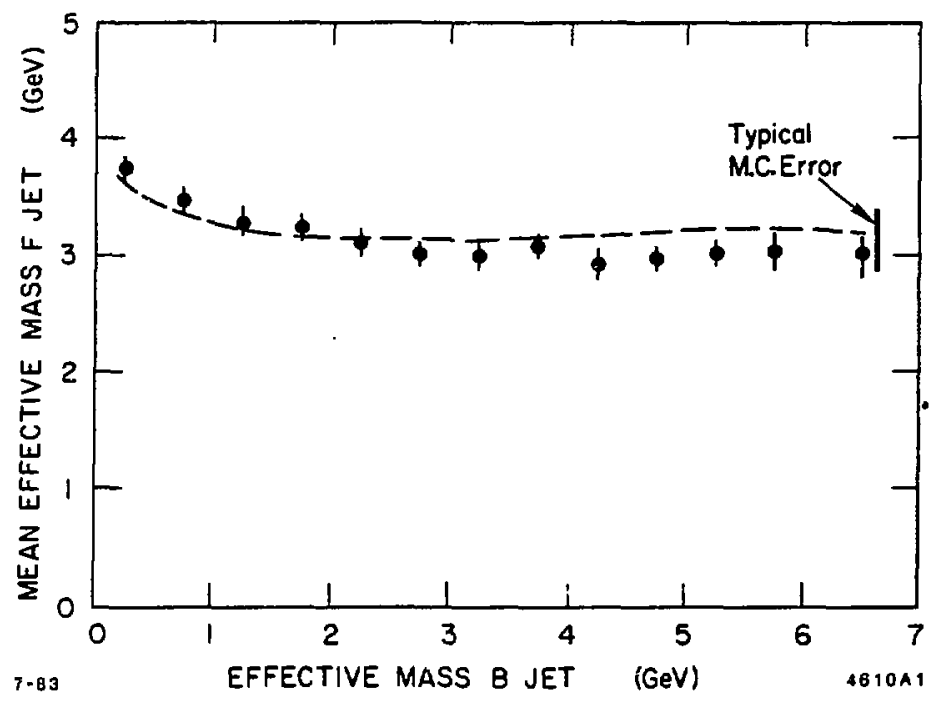

Fig. 6. Forward-backward jet mass correlations.

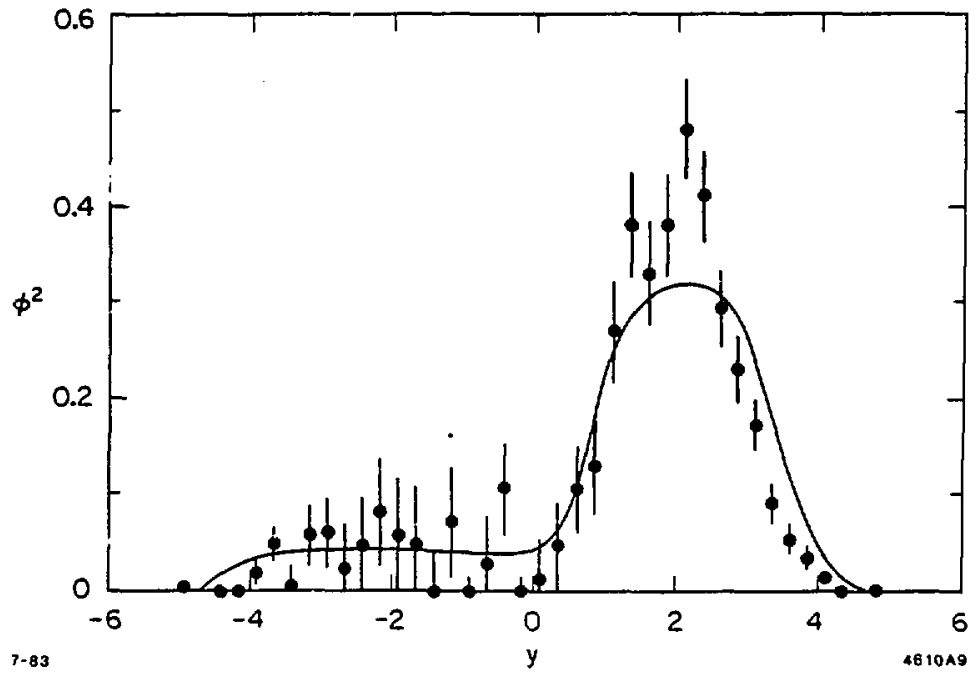

Fig. 7. Charge compensation test. The curve shows the Monte Carlo results. 


$$
\rho\left(y_{1}, y_{2}\right)=\frac{1}{\sigma} \frac{d^{2} \sigma}{d y_{1}, d y_{2}} \quad ; \quad \tilde{\phi}\left(y_{1}, y_{2}\right)=\frac{\phi\left(y_{1}, y_{2}\right)}{\int \phi\left(y_{1}, y_{2}\right) d y}
$$

The data show a predominant local charge compensation but, in addition, there is a finite correlation in the opposite jet with $y \leq 0$, such as would be expected if the jets originated from the fragmentation of oppositely charged quark pairs. As seen from the line, the Monte-Carlo events which come from charged quark fragmentation give a reasonable representation of the data. Similar results have already been reported by the TASSO collaboration ${ }^{14}$.

\subsection{CORRELATIONS IN RAPIDITY}

The early resuits from Fermila's') and the ISR ${ }^{2}$ ) showed that the particle produced exhibit short range clustering in rapidity. Recent $e^{+} e^{-}$data also show similar effects ${ }^{15}$ ).

The two body correlati a function that we use is:

$$
R=\frac{\rho_{2}\left(y_{1}, y_{2}\right)}{f \rho_{1}\left(y_{1}\right) \rho_{1}\left(y_{2}\right)}-1
$$

where

$$
\begin{aligned}
\rho_{2}\left(y_{1}, y_{2}\right)=\frac{1}{\sigma} \frac{d^{2} \sigma}{d y_{1} d y_{2}} & ; \quad \int \rho_{2}\left(y_{1}, y_{2}\right) d y_{1} d y_{2}=<n(n-1)> \\
\rho_{1}(y)=\frac{1}{\sigma} \frac{d \sigma}{d y} & ; \quad \int \rho_{1}(y) d y=<n>
\end{aligned}
$$

and

$$
f=\frac{\langle n(n-1)\rangle}{\langle n\rangle^{2}}
$$

Since $R$ is a ratio of density functions it is relatively free of experimental biasses.

The multiplicity dependence of $R^{c c}$ is shown in Fig. 8. The correlation strength, as measured by $R^{c c}$, is rather independent of multiplicity. Hadronic data at the ISR seem to show a decrease in the height of the correlation as the multiplicity increases ${ }^{16}$ ). It must be pointed out that the data of Ref. 16 are not relatively normalized $(f=1)$ while our data have $f=.892, .914$ and .955 for the three different bands of multiplicities studied. 


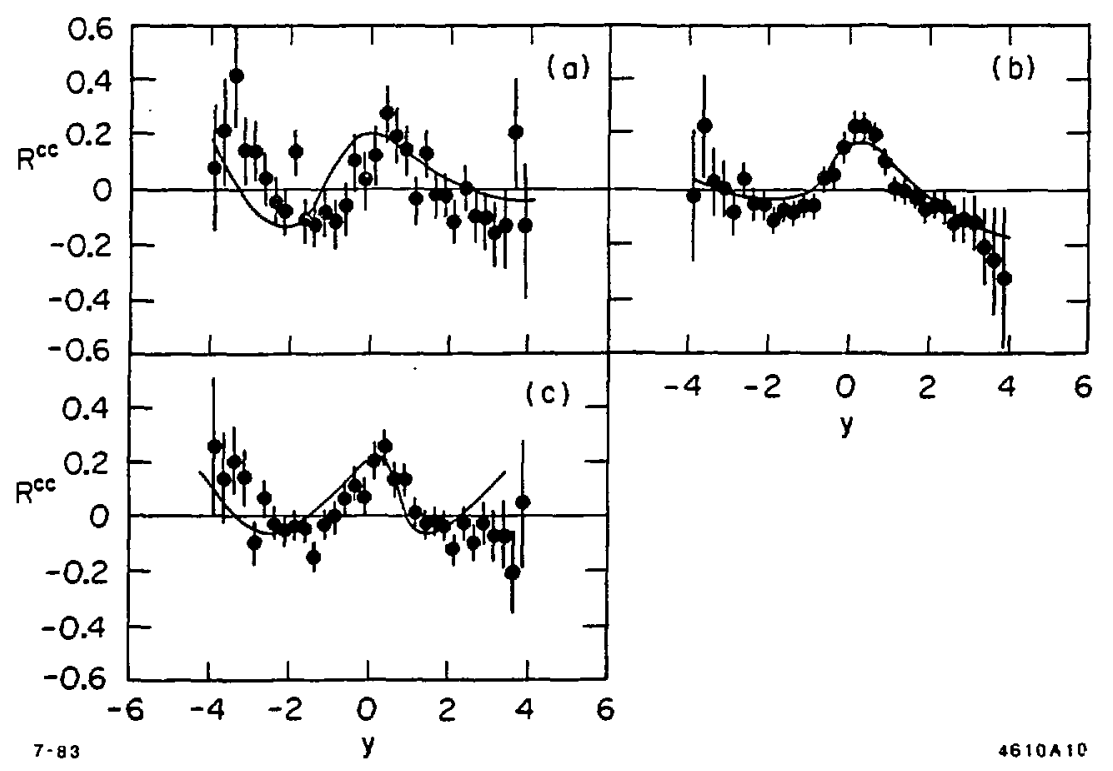

Fig. 8. Multiplicity dependence of central region charge-charge correlation $R^{c c}$ for (a) $5 \leq n_{c h} \leq 8$, (b) $10 \leq n_{c h} \leq 12$ and (c) $n_{c h} \leq 13$.

In Fig. 8 we compare our distribution of $R^{c c}$ for four different rapidity intervals with pp data analyzed by subtracting the leading protons ${ }^{17}$ ). The agreement is amazingly good. especially if contrasted with the same data without substraction of the leading particles ${ }^{16,17}$.

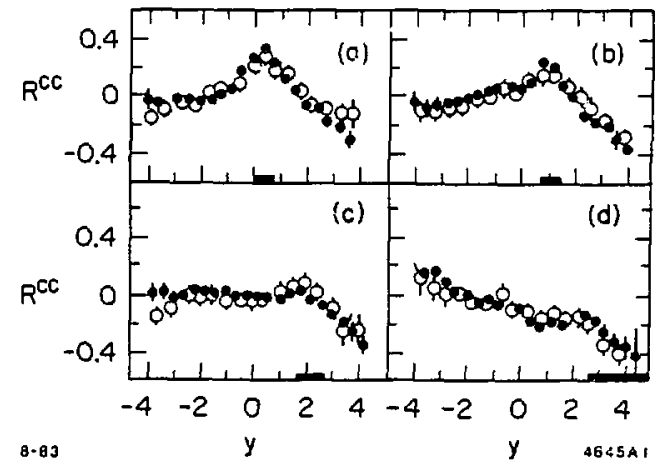

Fig. 8. Charge-charge rapidity correlation $R^{c c}$ for four selections of the test region (a) $0-.75, .75-1.5$, (c) $1.5-2.5$, and (d) 2.5 - 5.0. • HRS results; o, ref. 15. 
The short range correlation effect, observed in hadronic interactions, has been commonly explained in terms of cluster models. ${ }^{18)}$ An alternative method for studying particle clustering in rapidity is to use the rapidity gap between the charged particles. ${ }^{19)}$ If particles uniformly populate the full rapidity range $\left(y_{T}\right)$ available for a given multiplicity and the multiplicity distribution is Poisson-like with mean $\langle n\rangle$ then the density of gaps $(r)$ per unit of rapidity should be

$$
P(r)=\rho \exp (-\rho r)
$$

where

$$
\rho=\frac{\langle n\rangle}{y_{T}}
$$

Figure 10a shows the gap distribution of our two-jet sample. The line, which is the prediction of the above equation, with a slope of two, gives a good representation of the data. The rapidity distribution between negative particles shown in Fig. 10b also agrees with the above simple model, but now the slope in $1 / 2$ of that for the all-charged case.

It has been suggested ${ }^{19)}$ that the gap distribution at large gaps should characterize the cluster density rather than the pion density. Hadron data at Fermilab give some support to this idea. ${ }^{19)}$ Our $e^{+} e^{-}$data do not support this suggestion. Furthermore, since the slope is just equal to the pion rapidity density, the cluster multiplicity would be $\simeq 1$ charged pion on average.

\section{Conclusions}

Our data suggest that:

1. Partons fragment independently

2. Jets are initiated by charged quarks

3. Short range rapidity correlations are similar to those observed in hadron interactions qualitatively, but quantitative comparisions require careful work.

4. Cluster models do not seem to fit $e^{+} e^{-}$annihilation data. 


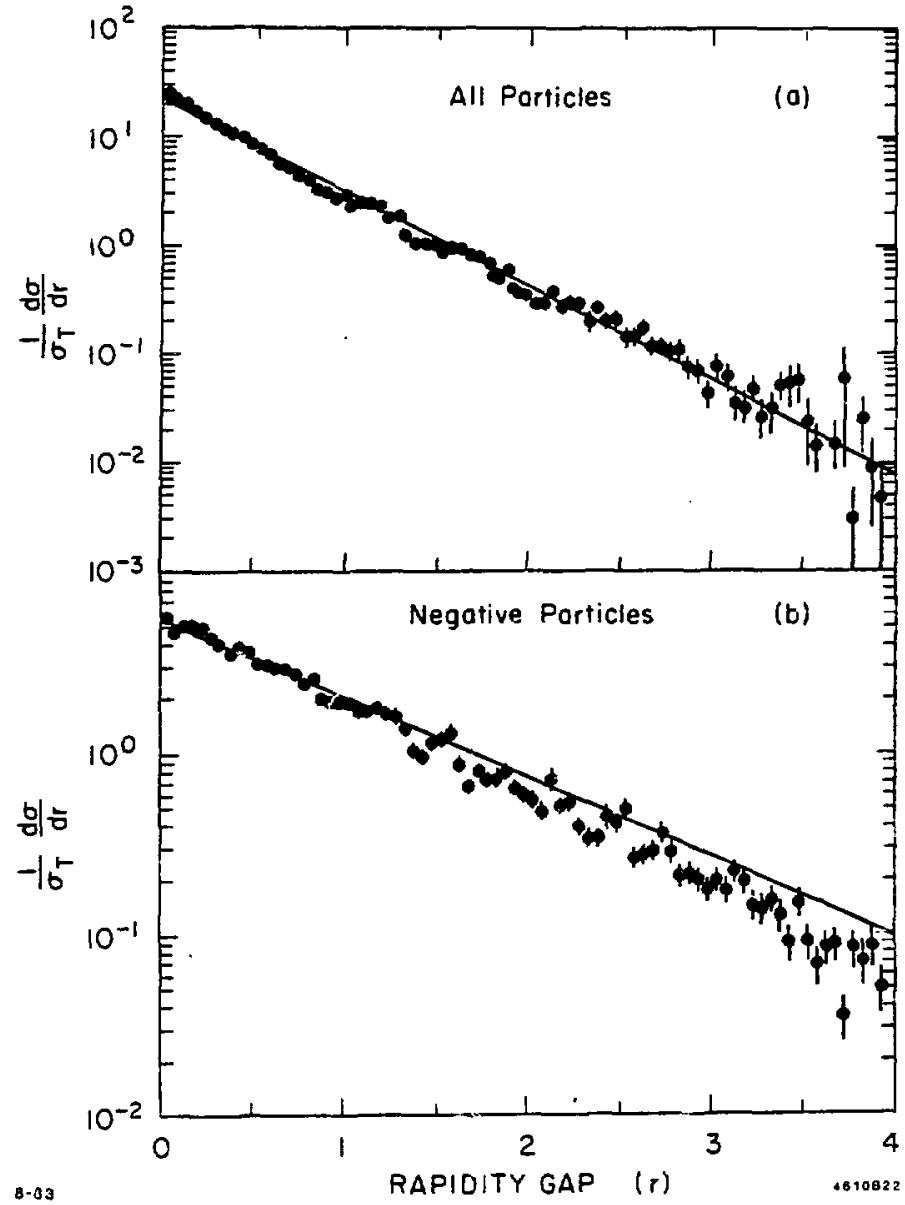

Fig. 10. (a) Rapidity gap distribution for all charged particles, (b) rapidity gap distribution for equal sign particles. The straight lines are the prediction of the simple model discussed in the text. 


\section{References}

1. J. Whitmore, Physics Reports 10, 273 (1974); 27, 187 (1976).

2. L. Foà ibid 22, (1975).

3. G. Arnison et al., Phys. Lett. 118B, 173 (1982); M. Banner, et al., ibid 118B, 203 (1982).

4. B. H. Wiik and G. Wolf, "Electron-Positron Interactions" Springer Tracts in Modern Physics Vol. 86.

5. P. B. Renton, Proceedings of XIII International Symposium on Multiparticle dynamics (1982).

6. D. J. Biorken and S. J. Brodsky, Phys. Rev. D $\underline{i}, 1416$ (1970).

7. B. Brandt et al., Phys. Lett. 12, 57 (1964); E. Fahri, Phys. Rev. Lett. 39, 1587 (1977).

8. Z. Koba et al., Nucl. Phys B40, 317 (1972).

9. C. Berger et al., DESY-Report 80/69 (1980).

10. P. Slattery, Phys. Rev. D $\underline{7}, 2073$ (1973).

1 J. Salava and V. Simak, Nucl. Phys. B69, 15 (1974).

12. P. D. Field and R. P. Feynman, Nucl. Phys. B136, 1 (1978).

13. S. Uhlig et al., Nucl. Phy:. B132, 15 (1978)

14. R. Brandelik et al., Phys. Lett. 100B, 357 (1981).

15. W. Koch Proceedings of XIII International Symposium on Multiparticle Dynamics DESY 82-072.

16. G. Giacomelli. University of Bologna Preprint IFUB 82/83 (1882).

17. J. Rerbiers et al. Letters al Nuovo Cimento $\underline{36}, 563$ (1983).

18. G. Giacomelli and M. Tacob Physics Reports $\underline{55}, 1$ (1979).

19. P. Yirila et al. Phys. Rev. D 12 , Q2 (1875). 